\title{
Light Output Enhancement of InGaN/GaN Light-Emitting Diodes with Contrasting Indium Tin-Oxide Nanopatterned Structures
}

\author{
Sang Hyun Jung, ${ }^{1,2}$ Keun Man Song, ${ }_{1}^{1}$ Young Su Choi, ${ }^{1}$ Hyeong-Ho Park, ${ }^{1}$ \\ Hyun-Beom Shin, ${ }^{1}$ Ho Kwan Kang, ${ }^{1}$ and Jaejin Lee $^{2}$ \\ ${ }^{1}$ Nano Process Division, Korea Advanced Nano Fab. Center, Suwon, Kyeonggi 443-270, Republic of Korea \\ ${ }^{2}$ Department of Electrical and Computer Engineering, Ajou University, Suwon, Kyeonggi 443-749, Republic of Korea
}

Correspondence should be addressed to Ho Kwan Kang; hokwan.kang@kanc.re.kr and Jaejin Lee; jaejin@ajou.ac.kr

Received 12 April 2013; Revised 9 July 2013; Accepted 9 July 2013

Academic Editor: M. Reza Bayati

Copyright (c) 2013 Sang Hyun Jung et al. This is an open access article distributed under the Creative Commons Attribution License, which permits unrestricted use, distribution, and reproduction in any medium, provided the original work is properly cited.

\begin{abstract}
Various nanopatterns on the transparent conducting indium tin oxide (ITO) layer are investigated to enhance the light extraction efficiency of the InGaN/GaN light-emitting diodes (LEDs). Triangular, square, and circular nanohole patterns with the square and hexagonal lattices are fabricated on the ITO layer by an electron beam lithography and inductively coupled plasma dry etching processes. The circular hole pattern with a hexagonal geometry is found to be the most effective among the studied structures. Light output intensity measurements reveal that the circular hole nanopatterned ITO LEDs with a hexagonal lattice show up to $35.6 \%$ enhancement of output intensity compared to the sample without nanopatterns.
\end{abstract}

\section{Introduction}

In recent years, InGaN/GaN light-emitting diodes (LEDs) have been used in many applications such as general white lightening, traffic signals, automobile head lights, and back light units of liquid crystal display [1]. Although InGaN/GaN LEDs are being commercially available, it is still necessary to further improve the light extraction efficiency and the internal quantum efficiency for the realization of the highefficiency and high-power LEDs. There are many methods to improve the efficiency of the InGaN/GaN LEDs such as substrate design, metal reflection layer, distributed Bragg reflector, omnidirectional reflector, truncated-inverted-pyramid chip geometry, nanopatterning, transparent substrate, surface texturing, and laser lift off [2-10]. In addition, the surface texturing or patterning of a p-type transparent conducting layer has been demonstrated to be effective for enhancing light extraction efficiency [11-18]. In the previous works, Pan et al. conducted photolithography and dry etching to fabricate the InGaN/GaN LEDs with a $3 \mu \mathrm{m}$ square surfacetextured ITO [15]. With the textured ITO surface, they achieved $16 \%$ output power enhancement compared to the planar ITO LEDs. Also, Chang et al. reported nitridebased LEDs with an ITO electrode, patterned by an imprint lithography and dry etching [11]. It was found that the LED output power for nanopatterned ITO LEDs with $0.85 \mu \mathrm{m}$ holes was about 12 and $8 \%$ higher than that of the planar ITO LEDs and micropatterned ITO LEDs with $1.75 \mu \mathrm{m}$ holes, respectively. Such a result suggested that submicron-sized holes can scatter light more effectively. It is thus worth investigating the nanopatterned structure and geometry on the ITO layer of the GaN-based LEDs for achieving the higher light extraction efficiency. Nanopatterning technique has attracted great attentions for more efficient light extraction from the LED structure and various methods are explored. Most of researches have been focused on the influences of periods and hole depths of the nanopatterns on the properties of the LEDs [11, 12, 18]. There are few studies on the light output enhancement by various geometry and shapes of the nanopatterned structure on the transparent conducting oxide layer of the LEDs. In this study, we report the light output analysis of the InGaN/GaN LEDs with varying geometric lattices and hole shapes fabricated on a transparent ITO layer. 


\section{Experiments}

GaN-based LED structures were grown on sapphire substrates by metalorganic vapor phase epitaxy (MOVPE, AIXTRON, G3 2600HT). For the growth of GaN, trimethylgallium (TMGa) and ammonia $\left(\mathrm{NH}_{3}\right)$ were used as the reactant sources for $\mathrm{Ga}$ and $\mathrm{N}$, respectively. Silane $\left(\mathrm{SiH}_{4}\right)$ and bis (cyclopentadienyl) magnesium $\left(\mathrm{Cp}_{2} \mathrm{Mg}\right.$ ) were used as $\mathrm{n}$ - and p-type dopants, respectively. A $30 \mathrm{~nm}$ thick $\mathrm{GaN}$ nucleation layer was grown on a sapphire substrate and followed by $2 \mu \mathrm{m}$ thick undoped GaN buffer, $3 \mu \mathrm{m}$ thick Si-doped n-GaN layer, InGaN/GaN multiple quantum wells (MQWs), and $0.15 \mu \mathrm{m}$ thick Mg-doped p-GaN layer. The MQWs region consists of $3 \mathrm{~nm}$ thick InGaN wells and $10 \mathrm{~nm}$ thick GaN barriers.

To fabricate $1 \mathrm{~mm}^{2}$ LED devices, mesa etching was carried out by a standard photolithography and inductively coupled plasma etching processes. A $200 \mathrm{~nm}$ thick ITO was deposited on top of the $\mathrm{p}-\mathrm{GaN}$ surface as a transparent conducting layer using an electron beam evaporator, followed by annealing at $550^{\circ} \mathrm{C}$ under the $\mathrm{N}_{2}$ flow for 4 min to obtain a good p-ohmic contact. After the deposition of $\mathrm{Cr} / \mathrm{Au}$ for an n-type contact, chip isolation was carried out by a standard photolithography and inductively coupled plasma etchings.

Triangular, square, and circular hole nanopatterns with the square and hexagonal lattices are generated by Layout Editor (L-EDIT, TANNER), which is the gds file generation program. To create the nanopatterns on the ITO layer, the GaN-based LED wafer was coated with 400 nm thick ZEP$520 \mathrm{~A}(\mathrm{ZEON})$, which is a positive-type electron beam resist. The square and hexagonal nanopatterned structures with various shaped holes including triangle, square, and circle were patterned using a $100 \mathrm{kV}$ electron beam lithography machine (JBX9300FS of JEOL). After the electron beam exposure, electron beam resist was developed by dipping in ZED-N50 (ZEON), rinsed in isopropyl alcohol (IPA), and blown with a $\mathrm{N}_{2}$ dry gun.

The samples then were etched by an inductively coupled plasma dry etcher (STS, MULTIPLEX LITE ICP System) using a $\mathrm{Cl}_{2} / \mathrm{CF}_{4}$ gas mixture. The electron beam resist on the etched LED wafer was removed by microwave asher (ULVAC, ENVIRO II) in the mixed $\mathrm{O}_{2}$ and $\mathrm{N}_{2} \mathrm{H}_{2}$ gas conditions. Figure 1(a) schematically describes the diagram of the LED chip structure with a patterned ITO surface. The microscopic image of the top view of the fabricated LED chip with a nanopatterned ITO is shown in Figure 1(b). The patterned samples were examined by a field emission scanning electron microscopy (FE-SEM, HITACHI, S-4800). Electroluminescence (EL), current-voltage $(I-V)$, and light output-current $(L-I)$ measurements were carried out using an in-line LED tester (WITHLIGHT, OPI-150). The diameter of the photodetector and the distance between the photodetector and LEDs are 11 and $60 \mathrm{~mm}$, respectively, in the EL measurement conditions.

\section{Results and Discussion}

The design parameters of the nanopatterns for an ITO layer are summarized in Table 1 . The shapes of the nanopatterns consist of triangle, square, and circle with either square or
TABLE 1: The lattice and dimension of the nanopatterns fabricated on the ITO layer by an electron beam lithography.

\begin{tabular}{|c|c|c|c|c|c|}
\hline Nanopatterns & Lattice & $\begin{array}{l}\text { Pattern } \\
\text { shape }\end{array}$ & $\begin{array}{c}X \text { pitch } \\
(\mathrm{nm})\end{array}$ & $\begin{array}{c}Y \text { pitch } \\
(\mathrm{nm})\end{array}$ & $\begin{array}{c}\text { Pattern } \\
\text { dimension } \\
(\mathrm{nm})\end{array}$ \\
\hline ST & \multirow{3}{*}{ Square } & Triangle & 400 & 400 & 304 \\
\hline SS & & Square & 400 & 400 & 200 \\
\hline SC & & Circle & 400 & 400 & 226 \\
\hline HT & \multirow{3}{*}{ Hexagonal } & Triangle & 400 & 693 & 283 \\
\hline HS & & Square & 400 & 693 & 186 \\
\hline $\mathrm{HC}$ & & Circle & 400 & 693 & 210 \\
\hline
\end{tabular}

hexagonal lattices for comparison study of shape and geometry dependencies on the InGaN/GaN LEDs performances. We designed six different types of nanopatterns, which are square lattice triangular (ST), square lattice square (SS), square lattice circular (SC), hexagonal lattice triangular (HT), hexagonal lattice square (HS), and hexagonal lattice circular hole (HC). The coverage of nanopatterned area is fixed at $25 \%$ by controlling the length and pitch of the nanopatterns. The dimensions of the triangular, square, and circular holes are 304,200 , and $226 \mathrm{~nm}$, respectively, with a $400 \mathrm{~nm}$ pitched square lattice. In case of the hexagonal lattice, $X$ and $Y$ pitches are 400 and $693 \mathrm{~nm}$, respectively, and the dimensions of the triangular, square, and circular holes are 283, 186, and $210 \mathrm{~nm}$, respectively. Scanning electron microscopy (SEM) images of the fabricated nanopatterns with varying hole-shapes and lattices are displayed in Figure 2. The etched depth of the ITO layer on the LEDs is $70 \mathrm{~nm}$.

Figure 3 shows the injection current versus voltage ( $I$ $V)$ characteristics of the nanopatterned ITO LEDs. Under $20 \mathrm{~mA}$ current injection, it was measured that forward voltage for the conventional ITO LED is $2.95 \mathrm{~V}$ and that of the nanopatterned ITO LED with the ST, SS, SC, HT, HS, and $\mathrm{HC}$ hole structures is 3.03, 3.02, 3.02, 3.00, 3.01, and $2.99 \mathrm{~V}$, respectively. It also reveals that the nanopatterned ITO electrode causes a slight increase of operating voltage. The slightly higher forward voltage could result from the dry etching damage to the ITO layer, which affects the sheet resistances and current spreading [13].

Figure 4 shows the light output versus injection current $(L-I)$ characteristics of the nanopatterned ITO LEDs. The output power increased with the injection current and no intensity saturation was observed up to $100 \mathrm{~mA}$ for all the nanopatterned LEDs. It was also found that the highest output power is achieved from the nanopatterned ITO LED with an HC hole structure. For the $20 \mathrm{~mA}$ injection current, the LED output powers are 3.25, 3.61, 3.87, 4.03, $3.77,4.03$, and $4.40 \mathrm{~mW}$ for the conventional ITO LED, nanopatterned ITO LED with the ST, SS, SC, HT, HS, and $\mathrm{HC}$ hole structures, respectively. Table 2 summarizes the relative light output intensity increments of the samples with the different nanopatterns. The light output intensities of the nanopatterned ITO LEDs with the ST, SS, SC, HT, HS, and $\mathrm{HC}$ hole structures increase by 11.0, 19.1, 24.2, 16.2, 24.1 , and $35.6 \%$, respectively, compared to the one without 


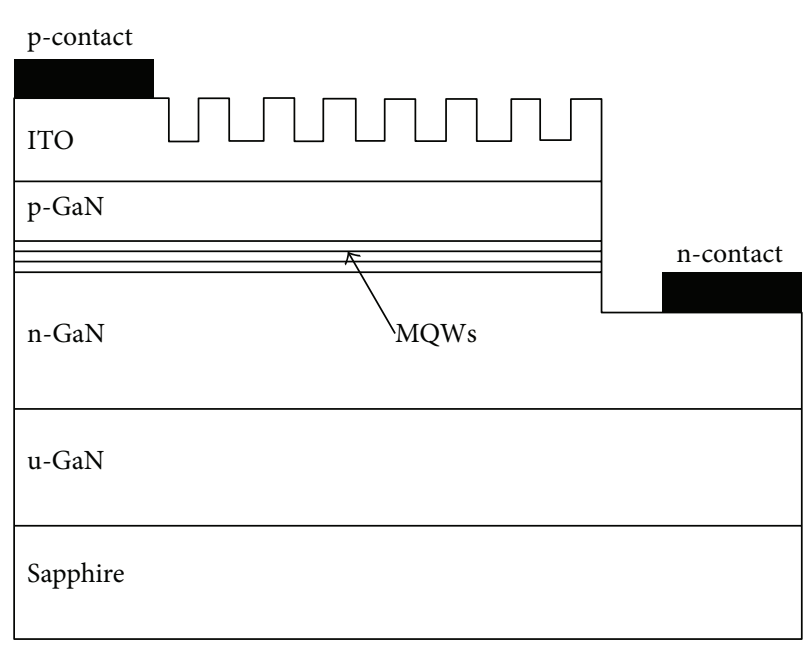

(a)

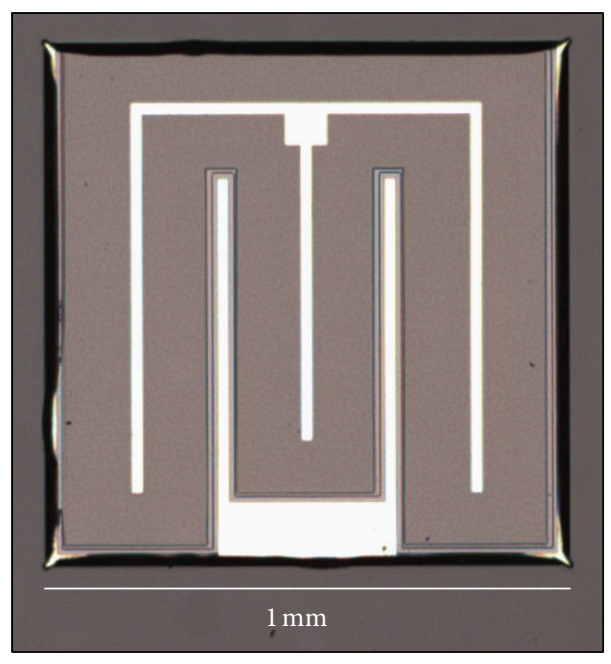

(b)

FIGURE 1: (a) Schematic diagram of the InGaN/GaN LED structure and (b) top-view microscopic image of the fabricated ITO LED chip with nanopatterns.
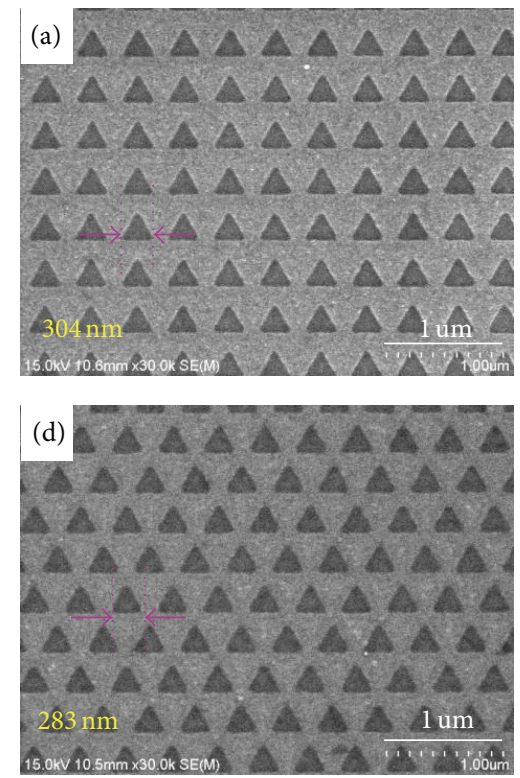
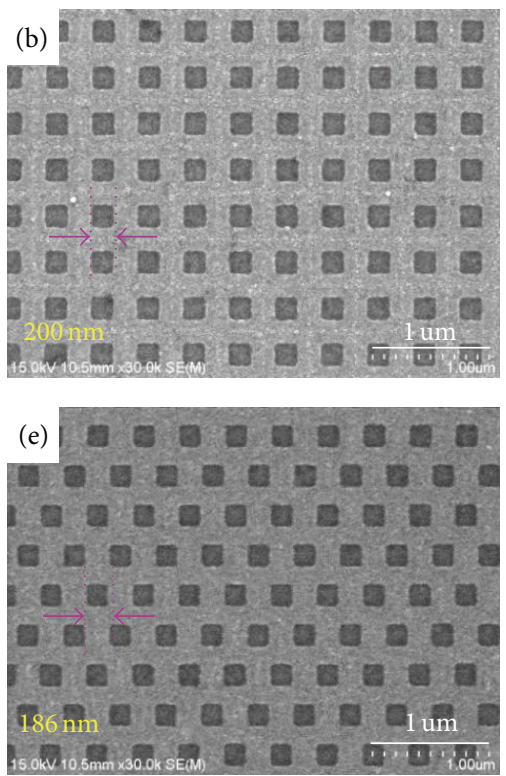
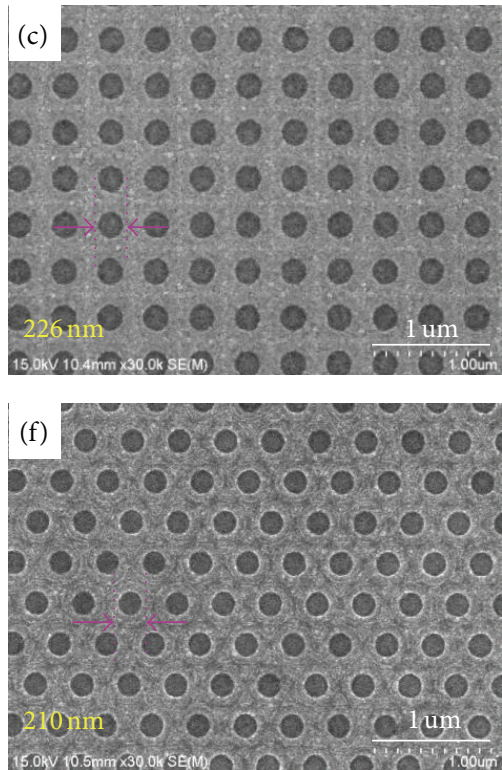

FIGURE 2: SEM images of the (a) triangular, (b) square, and (c) circular holes with the square lattice and (d) triangular, (e) square, and (f) circular holes with the hexagonal lattice on the ITO layer.

the nanopatterns. According to Snell's law, the critical angle of the total internal reflection at air/ITO interface is around $29^{\circ}$, assuming the refractive index of the ITO to be 2.06 at $437 \mathrm{~nm}$ wavelength. Thus, a significant amount of photon will be reflected at the sample surface for conventional ITO LEDs. The nanopatterned ITO layer results in the formation of large sidewalls, with which some of the reflected light may escape through the sidewall air/ITO interfaces, resulting in additional light extraction and, in turn, enhancement of the light output power. The more efficient relative light output intensity in the nanopatterned LEDs may be attributed to the improvement of the spontaneous Purcell emission rate [7].
TABLE 2: The relative light output increment of the InGaN/GaN LEDs with a nanopatterned ITO layer.

\begin{tabular}{lccc}
\hline \multirow{2}{*}{$\begin{array}{l}\text { Lattice type } \\
\end{array}$} & \multicolumn{3}{c}{ Relative increment of light output intensity (\%) } \\
Triangular holes & Square holes & Circular holes \\
\hline Square & 11.0 & 19.1 & 24.2 \\
Hexagonal & 16.2 & 24.1 & 35.6 \\
\hline
\end{tabular}

As shown in Figure 4 and Table 2, circular hole pattern on the ITO layer is measured to be more effective in enhancing the light extraction efficiency than the square and triangular 


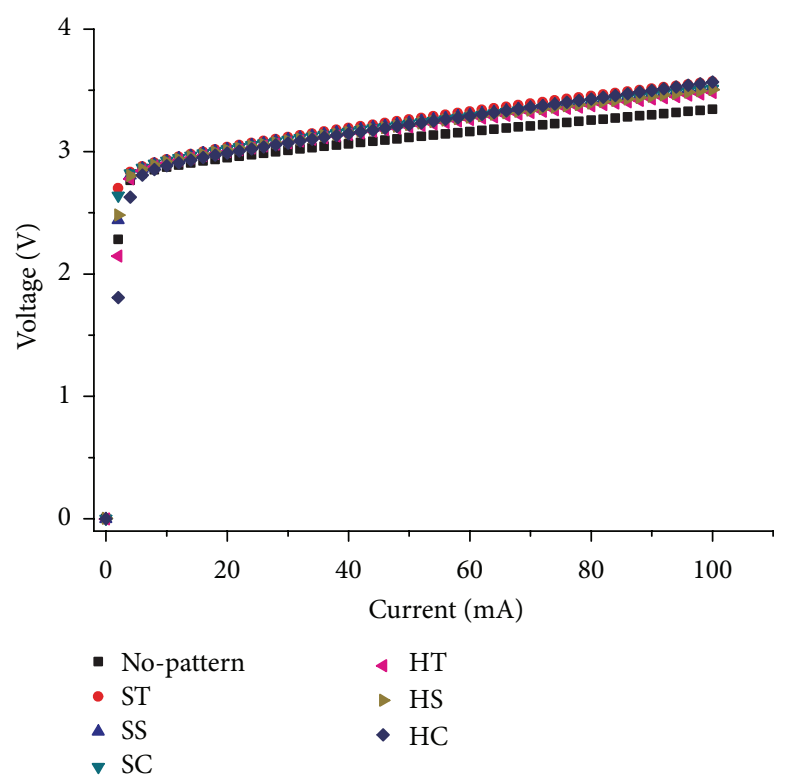

FIGURE 3: Voltage versus current characteristics of the nanopatterned ITO LEDs at room temperature.

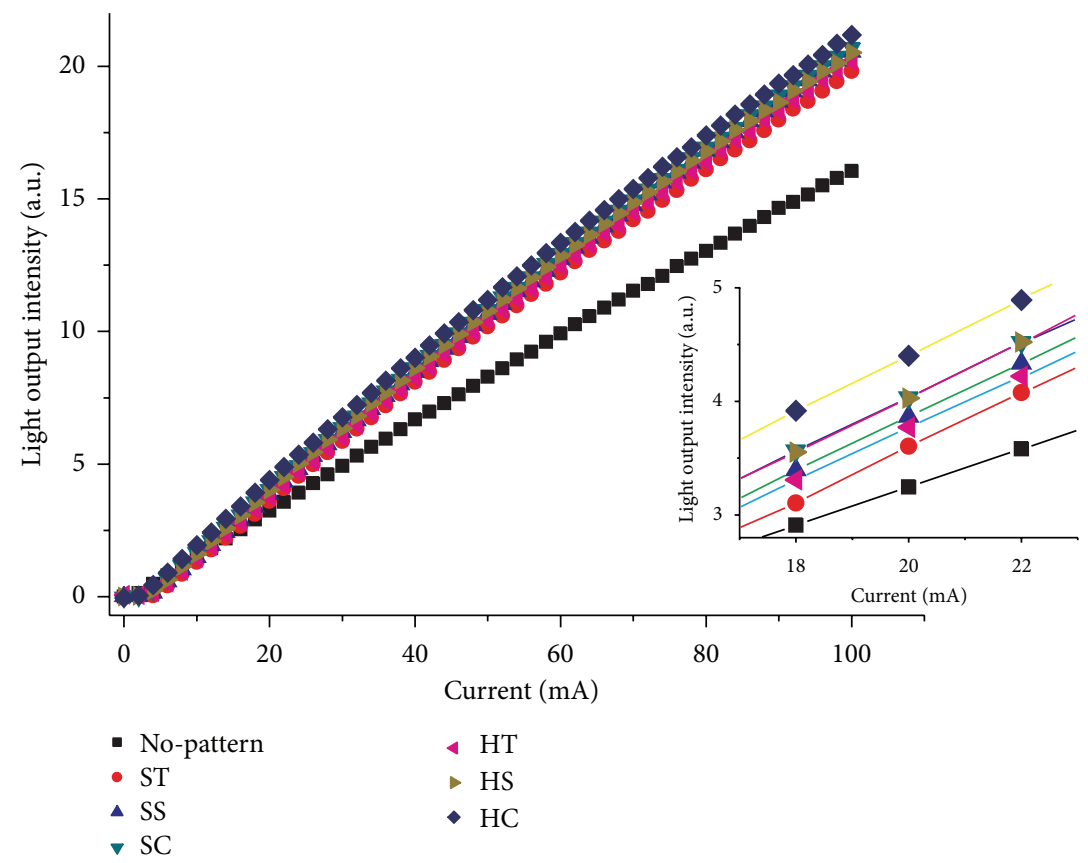

FIGURE 4: Light output versus injection current $(L-I)$ characteristics of the nanopatterned ITO LEDs at room temperature. The inset shows light output versus injection current $(L-I)$ characteristics at $20 \mathrm{~mA}$ injection current.

hole-patterns. Photons generated in the multiple quantum well (MQW) regions may escape more efficiently at the ITO/air sidewall interfaces through the circular hole patterns due to the unfolded side surface areas. Furthermore, the light output of the LEDs with the nanopatterned ITO with a hexagonal lattice is greater than those with the square lattice because the number of hole patterns per unit ITO area of hexagonal lattice is higher than that of square lattice.

Figure 5 shows the room temperature EL spectra of the nanopatterned ITO LED at $20 \mathrm{~mA}$ injection current. EL intensities of the LEDs with the nanopatterned ITO layer are measured to be significantly increased compared to the one without the nanopatterns. The EL intensity of the nanopatterned ITO LEDs with the HC hole structures increases by $35.6 \%$ compared to the one without the nanopatterns. Our results for the HC structures with $0.21 \mu \mathrm{m}$ holes show better EL intensity enhancement compared to the previously reported 12\% EL enhancement from the nanopatterned ITO LEDs with $0.85 \mu \mathrm{m}$ holes [11]. It could be attributed to the increment of the number of hole patterns per unit ITO area by 


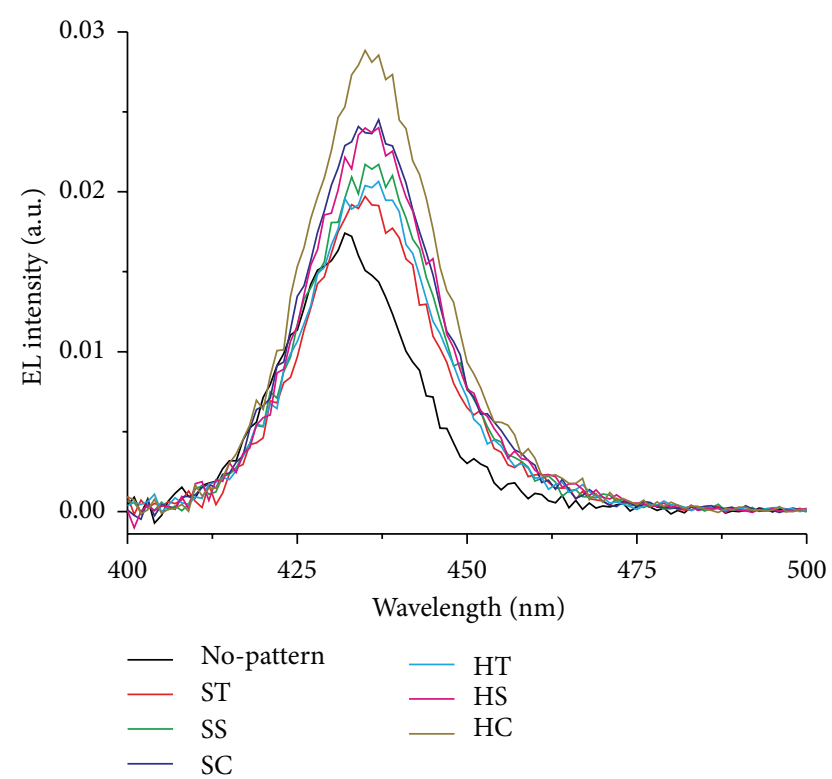

FIGURE 5: EL spectra of the nanopatterned ITO LEDs at room temperature.

the smaller hole pattern, which scatters light more efficiently than the larger hole pattern. The circular nanopatterned ITO LEDs with the hexagonal lattice show the highest enhancement of the EL intensity, consistent with the results from the light output intensity current measurements. The possibility of vertical penetration of photons at the ITO/air hole interfaces is enhanced by the increased surface areas of the circular hole patterns in comparison with the other square and triangular hole patterns. Furthermore, the EL intensity of the LEDs with the nanopatterned ITO with a hexagonal lattice is greater than those with the square lattice. Because the number of hole patterns per unit ITO area of the hexagonal lattice is larger than that of the square lattice, the circular nanopatterned ITO LEDs with the hexagonal lattice show the greatest EL intensity enhancement. The main EL emission peak of the nanopatterned ITO LEDs is measured to be red-shifted; for example, the main peak emission for the nanopatterned ITO LEDs with an HC hole structure was observed at $437 \mathrm{~nm}$, while that of the conventional ITO LEDs was measured at $432 \mathrm{~nm}$. It is known that the main EL emission peak of the InGaN/GaN LEDs with a nanopatterned ITO layer shifts to a longer wavelength compared to the nonpatterned ITO LEDs due to the Joule heating effect [19]. This could be attributed to the increased surface areas of the patterned ITO layer, resulting in more heat dissipation through the increased surface.

\section{Conclusions}

Various nanopatterns have been fabricated on the transparent conducting ITO layer of the InGaN/GaN LEDs by the electron beam lithography and inductively coupled plasma dry etching process. It is found that the InGaN/GaN LEDs fabricated with the nanopatterned ITO layer produce a higher light output power than the sample with the planar ITO electrodes. The circular hole pattern with a hexagonal geometry is found to be the most effective among the tested structures. The light output intensity of the hexagonal lattice with the circular hole patterns on the ITO layer of the InGaN/GaN LED showed an enhancement of $35.6 \%$ over that of the conventional LED with a planar ITO at $20 \mathrm{~mA}$ injection current.

\section{Conflict of Interests}

The authors declare no competing financial interests.

\section{Acknowledgment}

This work is supported by the National Research Foundation Grants (Midcareer Researcher Program: 2009-0083803 and KRF-2007-331-D00250).

\section{References}

[1] N. Narendran, Y. Gu, J. P. Freyssinier-Nova, and Y. Zhu, "Extracting phosphor-scattered photons to improve white LED efficiency," Physica Status Solidi A, vol. 202, no. 6, pp. R60-R62, 2005.

[2] T. Nishida, H. Saito, and N. Kobayashi, "Efficient and highpower AlGaN-based ultraviolet light-emitting diode grown on bulk GaN," Applied Physics Letters, vol. 79, no. 6, pp. 711-712, 2001.

[3] T. Gessmann and E. F. Schubert, "High-efficiency AlGaInP light-emitting diodes for solid-state lighting applications," Journal of Applied Physics, vol. 95, no. 5, pp. 2203-2216, 2004.

[4] D. A. Steigerwald, J. C. Bhat, D. Collins et al., "Illumination with solid state lighting technology," IEEE Journal on Selected Topics in Quantum Electronics, vol. 8, no. 2, pp. 310-320, 2002.

[5] C.-F. Chu, F.-I. Lai, J.-T. Chu et al., "Study of GaN light-emitting diodes fabricated by laser lift-off technique," Journal of Applied Physics, vol. 95, no. 8, pp. 3916-3922, 2004.

[6] S. Noda, M. Fujita, and T. Asano, "Spontaneous-emission control by photonic crystals and nanocavities," Nature Photonics, vol. 1, no. 8, pp. 449-458, 2007.

[7] T. N. Oder, J. Shakya, J. Y. Lin, and H. X. Jiang, "III-nitride photonic crystals," Applied Physics Letters, vol. 83, no. 6, pp. 12311233, 2003.

[8] T. N. Oder, K. H. Kim, J. Y. Lin, and H. X. Jiang, "III-nitride blue and ultraviolet photonic crystal light emitting diodes," Applied Physics Letters, vol. 84, no. 4, pp. 466-468, 2004.

[9] J. J. Wierer, M. R. Krames, J. E. Epler et al., "InGaN/GaN quantum-well heterostructure light-emitting diodes employing photonic crystal structures," Applied Physics Letters, vol. 84, no. 19, pp. 3885-3887, 2004.

[10] S.-F. Yu, S.-P. Chang, S.-J. Chang, R.-M. Lin, H.-H. Wu, and W.C. Hsu, "Characteristics of InGaN-based light-emitting diodes on patterned sapphire Substrates with various pattern heights," Journal of Nanomaterials, vol. 2012, Article ID 346915, 6 pages, 2012.

[11] S. J. Chang, C. F. Shen, W. S. Chen et al., "Nitride-based light emitting diodes with indium tin oxide electrode patterned by imprint lithography," Applied Physics Letters, vol. 91, no. 1, Article ID 013504, 2007.

[12] X. Zhang, S. Liu, Y. Liu, X. Chen, H. Lin, and X. Ren, "Enhancement of LED light extraction via diffraction of hexagonal lattice 
fabricated in ITO layer with holographic lithography and wet etching," Physics Letters A, vol. 372, no. 20, pp. 3738-3740, 2008.

[13] D.-S. Leem, T. Lee, and T.-Y. Seong, "Enhancement of the light output of GaN-based light-emitting diodes with surfacepatterned ITO electrodes by maskless wet-etching," Solid-State Electronics, vol. 51, no. 5, pp. 793-796, 2007.

[14] M. Yamada, T. Mitani, Y. Narukawa et al., "InGaN-based nearultraviolet and blue-light-emitting diodes with high external quantum efficiency using a patterned sapphire substrate and a mesh electrode," Japanese Journal of Applied Physics, vol. 41, no. 2, pp. L1431-L1433, 2002.

[15] S.-M. Pan, R.-C. Tu, Y.-M. Fan, R.-C. Yeh, and J.-T. Hsu, "Improvement of InGaN-GaN light-emitting diodes with surfacetextured indium-tin-oxide transparent ohmic contacts," IEEE Photonics Technology Letters, vol. 15, no. 5, pp. 649-651, 2003.

[16] P. Uthirakumar, J. H. Kang, B. D. Ryu, H. G. Kim, H. K. Kim, and C.-H. Hong, "Nanoscale ITO/ZnO layer-texturing for highefficiency InGaN/GaN light emitting diodes," Materials Science and Engineering B, vol. 166, no. 3, pp. 230-234, 2010.

[17] D.-S. Leem, T. Lee, and T.-Y. Seong, "Enhancement of the light output of GaN-based light-emitting diodes with surfacepatterned ITO electrodes by maskless wet-etching," Solid-State Electronics, vol. 51, no. 5, pp. 793-796, 2007.

[18] K.-M. Yoon, K.-Y. Yang, K.-J. Byeon, and H. Lee, "Enhancement of light extraction in GaN based LED structures using $\mathrm{TiO} 2$ nano-structures," Solid-State Electronics, vol. 54, no. 4, pp. 484487, 2010.

[19] X. A. Cao and S. D. Arthur, "High-power and reliable operation of vertical light-emitting diodes on bulk GaN," Applied Physics Letters, vol. 85, no. 18, pp. 3971-3973, 2004. 

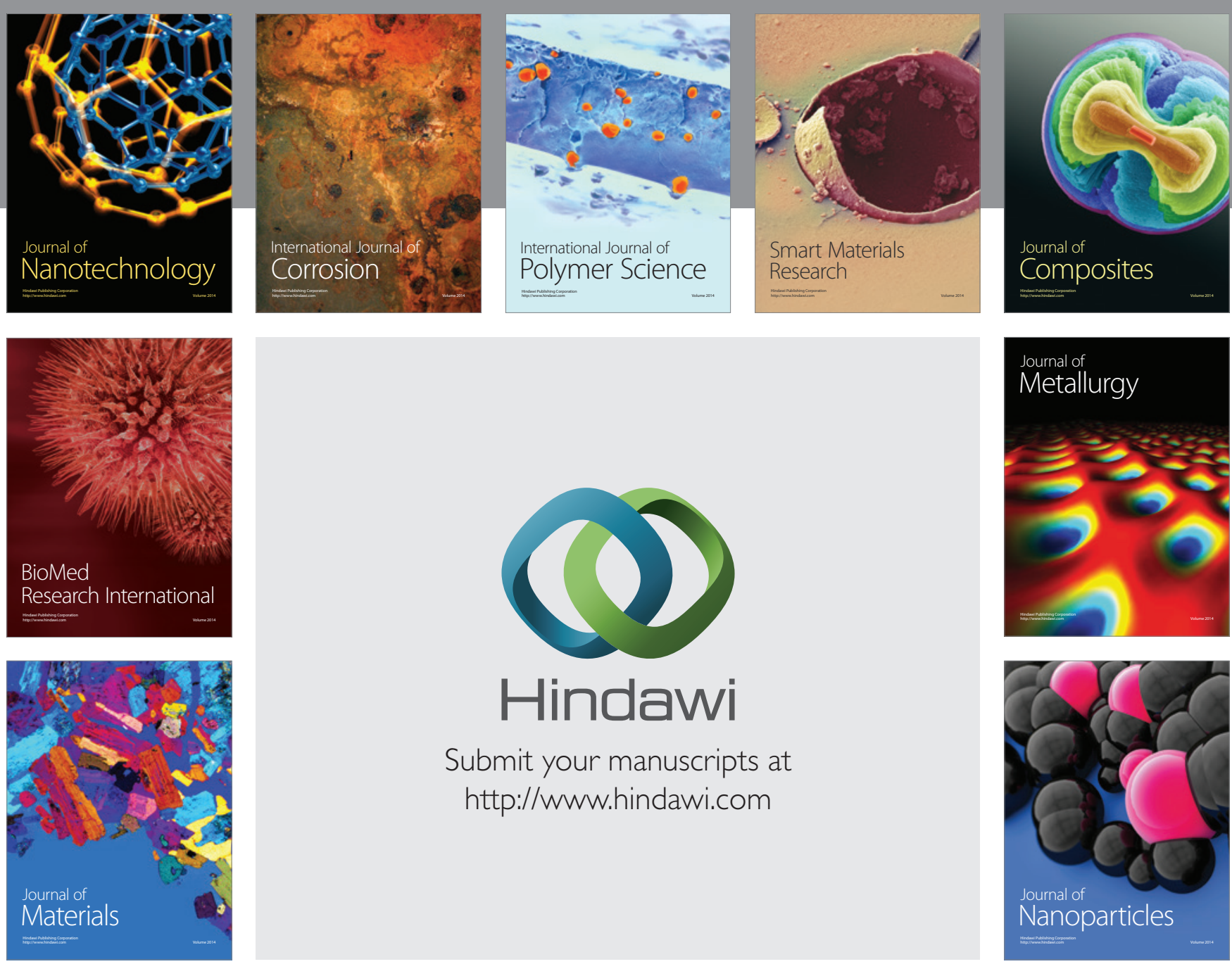

Submit your manuscripts at http://www.hindawi.com
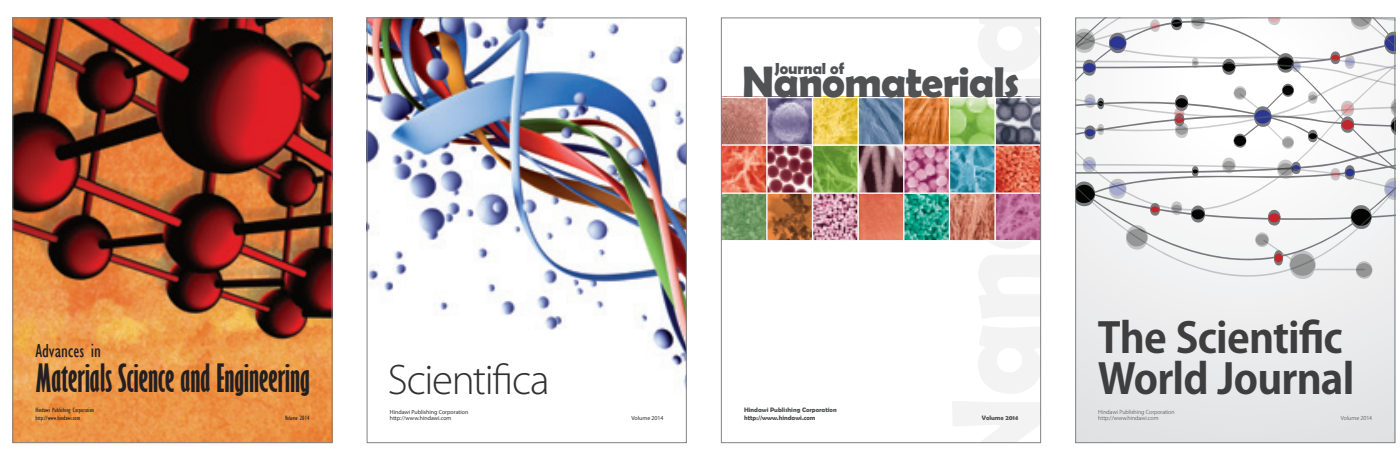

\section{The Scientific World Journal}
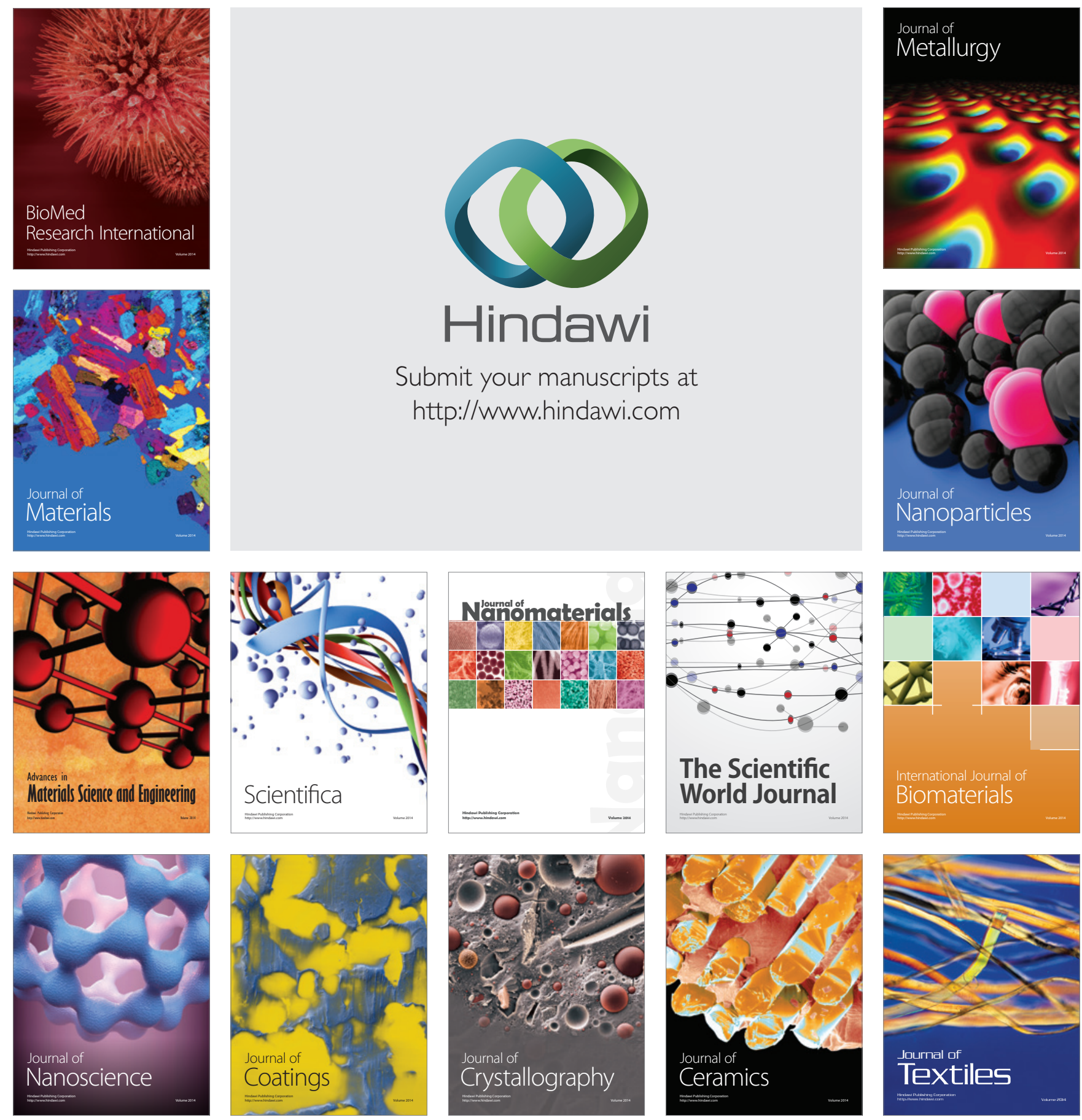